\title{
artigo
}

\section{Avaliação de maternidades: uma revisão integrativa da literatura}

Maternity assessment: an integrative literature review

Evaluación de la maternidad: revisión integradora de la literatura

\section{RESUMO}

Objetivo: Analisar a produção científica sobre a avaliação das maternidades incluindo elementos de estrutura, processos e resultados. Métodos: revisão integrativa da literatura a partir das bases MEDLINE, SciELO, CINAHL e LILACS entre os anos de 2008 e 2018. Os descritores utilizados foram: delivery rooms; maternal and child health; hospital maternity; birthing centers; program evaluation; health services; outcome assessment (health care). Resultados: A amostra final foi constituída por 17 artigos científicos. A maioria dos estudos atribuíram a estrutura como forma de avaliação por meio de domínios como infraestrutura adequada, disponibilidade de equipamentos, medicamentos e recursos humanos capacitados e avaliaram ambientes neonatais. Em segundo lugar, a qualidade das maternidades foi atribuída à satisfação das mulheres. Conclusão: indicadores de estrutura, processos e resultados são elementos constitutivos da avaliação em saúde nas maternidades, que podem ser avaliados de forma multidimensional e considerar a satisfação das mulheres.

DESCRITORES: Saúde Materno-Infantil; Maternidades; Salas de Parto; Centros de Assistência à Gravidez e ao Parto; Avaliação de Programas e Projetos de Saúde.

\section{ABSTRACT}

Objective: To analyze a scientific production on the evaluation of maternity hospitals including elements of structure, processes and results. Methods: integrative literature review based on MEDLINE, SciELO, CINAHL and LILACS between 2008 and 2018. The descriptors used were: delivery rooms; maternal and child health; hospital maternity; birth centers; program evaluation; health services; outcome assessment (health care).Results: The final sample consisted of 17 scientific articles. Most studies have assigned a structure as a form of assessment through domains such as availability, availability of equipment, medicines and trained human resources and assessment of neonatal environments. Second, the quality of maternity hospitals was attributed to women's satisfaction. Conclusion: indicators of structure, processes and results are constitutive elements of health assessment in maternity hospitals, which can be taken in a multidimensional way and consider women's satisfaction.

DESCRIPTORS: Maternal and Child Health; Maternity; Delivery Rooms; Birthing Centers; Program Evaluation.

\section{RESUMEN}

Objetivo: analizar una producción científica sobre la evaluación de las maternidades incluyendo elementos de estructura, procesos y resultados. Métodos: revisión integradora de la literatura basada en las bases de datos MEDLINE, SciELO, CINAHL y LILACS entre 2008 y 2018. Los descriptores utilizados fueron: salas de parto; Salud maternal e infantil; hospital de Maternidad; centros de maternidad; Programa de Evaluación; servicios de salud; evaluación de resultados (atención médica). Resultados: La muestra final estuvo compuesta por 17 artículos científicos. La mayoría de los estudios han asignado una estructura como forma de evaluación a través de dominios como disponibilidad, disponibilidad de equipos, medicamentos y recursos humanos capacitados y evaluación de ambientes neonatales. En segundo lugar, la calidad de las maternidades se atribuyó a la satisfacción de las mujeres. Conclusión: los indicadores de estructura, procesos y resultados son elementos constitutivos de la evaluación de la salud en las maternidades, que se pueden tomar de forma multidimensional y considerar la satisfacción de las mujeres.

DESCRIPTORES: Salud Materno-Infantil; Maternidades; Salas de partos; Centros de asistencia al embarazo y al parto; Evaluación de Programas y Proyectos de Salud.

RECEBIDO EM: 27/01/2021 APROVADO EM: 18/02/2021 


\section{Juliano Teixeira Moraes}

Enfermeiro Estomaterapeuta, Doutor. Professor Adjunto do Curso de Enfermagem - Universidade Federal de São João del-Rei - Campus Centro Oeste, Divinópolis, Minas Gerais, Brasil.

ORCID: 0000-0002-1109-962X

\section{Mateus Oliveira Marcelino}

Enfermeiro. Mestre em Enfermagem pela Universidade Federal de São João del-Rei - Campus Centro Oeste, Divinópolis, Minas Gerais, Brasil.

ORCID: 0000-0001-6484-995x

\section{Rafaela das Graças Santiago Faria}

Enfermeira. Mestranda em Enfermagem pela Universidade Federal de São João del-Rei - Campus Centro Oeste, Divinópolis, Minas Gerais, Brasil.

ORCID: 0000-0001-9508-8372

\section{INTRODUÇÃO}

A té o século XVIII, o parto envolvia altos índices de morbimortalidade materna e fetal, então a prática de cuidados com a saúde da mulher começou a se organizar. A partir do século XIX, a medicina desenvolveu técnicas cirúrgicas e anestésicas capazes de combater os altos índices de infecção puerperal, tornando o parto um evento hospitalar. Desde então, novas técnicas surgiram e foram aprimoradas e o parto hospitalar foi incorporado culturalmente pela sociedade $^{(1)}$.

No Brasil, a sua institucionalização foi integralmente expressiva somente após a Segunda Guerra Mundial. Tal fato trouxe queda considerável na mortalidade infantil, o que foi fator importante para que houvesse forte adesão e aceitação da hospitalização. Atualmente, ainda que as taxas de cobertura hospitalar ao parto sejam altas, o país enfrenta problemas de acesso, falta de estrutura física e recursos humanos capacitados, além de uma evidente desorganização na distribuição de leitos obstétricos e neonatais ${ }^{(2)}$.

Com os avanços na saúde materno infantil nas últimas décadas, observaram-se melhoras dos índices de saúde graças a esforços governamentais e de movimentos sociais. A mortalidade infantil reduziu drasticamente devido a ações desenvolvidas no componente pós-neonatal, mas com desafios persistentes no componente neonatal. As evidências demonstram que a maioria desses óbitos, principalmente ma-
A mortalidade infantil reduziu drasticamente devido a ações desenvolvidas

no componente

pós-neonatal,

mas com desafios

persistentes no

componente

neonatal. As

evidências

demonstram que a

maioria desses óbitos,

principalmente

maternos, são

evitáveis e acontecem

dentro dos hospitais. ternos, são evitáveis e acontecem dentro dos hospitais ${ }^{(3)}$.

Além do fato das estruturas hospitalares mais avançadas se concentrarem nas capitais, muitas ainda apresentam fragilidade na disposição de ferramentas e equipamentos para uma assistência de qualidade durante o trabalho de parto e nascimento. Entretanto, não se pode atribuir às taxas, ainda elevadas, de mortalidade na saúde materno-fetal somente à custa da estrutura hospitalar. Faz-se necessário ampliar o espectro ao nível de atendimento oferecido durante todo o processo de gestação, parto e puerpério por parte da equipe obstétrica ${ }^{(4)}$.

Contudo, vale ressaltar que, independente do risco gestacional, as gestantes devem ser acompanhadas por profissionais qualitativamente capacitados. O papel do profissional de saúde é garantir o monitoramento materno e fetal, intervir quando necessário e referenciar a gestante para níveis mais complexos quando indicado. As mulheres buscam locais seguros para terem seus filhos vivos e se direcionam para locais onde prezam pelo respeito, profissionais habilitados e estrutura adequada. Pode-se afirmar, portanto, que elementos da estrutura e processos repercutem diretamente na ocorrência de óbitos maternos e perinatais $^{(5)}$.

O objetivo dessa pesquisa foi, portanto, analisar a produção científica em relação à avaliação das maternidades, nos aspectos da estrutura, dos processos e dos resultados. 


\section{artigo}

\section{MÉTODOS}

Foi realizada uma revisão integrativa com base na literatura científica sobre a avaliação de maternidades em todo o mundo entre os anos de 2008 e 2018 nas bases de dados MEDLINE, SciELO, LILACS e CINAHL. Para elaboração do estudo, foram percorridas seis etapas: identificação da hipótese ou questão de pesquisa para a elaboração da revisão integrativa; estabelecimento de critérios para inclusão e exclusão de estudos ou amostragem ou busca na literatura; definição das informações a serem extraídas dos estudos selecionados ou categorização de estudos; avaliação dos estudos incluídos na revisão integrativa; interpretação dos resultados; apresentação da revisão ou síntese do conhecimento.

Os dados foram coletados entre novembro e dezembro de 2018 e foi delineado um panorama de dez anos para a realização das buscas. Incluíram-se estudos que abordam a avaliação de serviços de atendimento a mulheres e recém-nascidos no parto e nascimento, publicações na íntegra disponíveis nos idiomas português, inglês e espanhol. Foram excluídas teses, disser- tações, trabalhos publicados em anais de eventos, duplicatas e artigos disponíveis somente em resumo.

A pergunta de pesquisa foi estruturada da seguinte forma: como estão sendo abordados os aspectos estruturais, de processos e de resultados nas avaliações das maternidades nas investigações científicas?

Para as bases MEDLINE, SciELO, LILACS e CINAHL foram utilizados os termos: "Delivery rooms" OR "salas de parto" OR "salas de parto" OR "maternal and child health" OR "saúde materno-infantil" OR "salud materno-infantil" OR "hospital maternity" OR "maternidades" OR "maternidades" OR "birthing centers" $\mathrm{OR}$ "centros de assistência à gravidez e ao parto" OR "centros de assistencia al embarazo y al parto" AND "program evaluation" OR "avaliação de programas e projetos de saúde" OR "evaluacion de programas y proyectos de salud" AND "health services" OR "serviços de saúde" OR "servicios de salud" AND "outcome assessment (health care)" OR "avaliação de resultados (cuidados de saúde)" OR "evaluacion de resultado (atención de salud)".

A amostra inicial constituiu-se de 9.340 artigos, sendo 7.719 (MEDLINE);

Figura 1 - Fluxograma de identificação, seleção e inclusão dos estudos (PRISMA). Divinópolis, MG, Brasil, 2020.

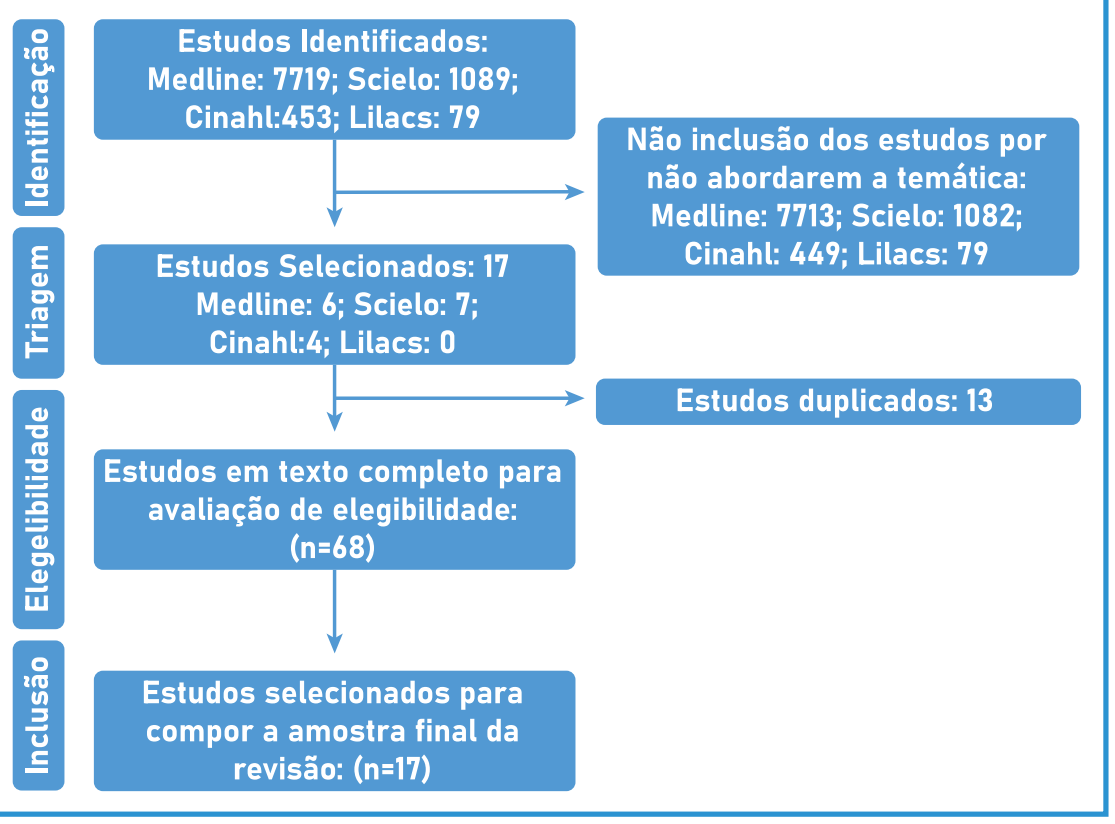

1.089 (SciELO); 453 (CINAHL) e 79 (LILACS).

A seleção dos artigos foi realizada de forma independente por dois revisores e conduzida por meio do protocolo Preferred Reportingn Items for Systematics Reviews and Meta-Analyses (PRISMA), conforme apresentado no fluxograma (Figura 1) de seleção dos estudos. Após leitura de títulos e resumos foram eleitos 68 estudos para leitura completa. As discordâncias entre os dois revisores foram esclarecidas por um terceiro revisor. Um instrumento metodológico foi criado pelos próprios autores para extração das seguintes informações: identificação do estudo, autores, fonte de informação, periódico, ano de publicação, objetivo, tipo de estudo, amostra estudada, categoria do estudo e nível de evidência, método e variáveis utilizadas.

Dessa forma, os estudos selecionados foram classificados conforme os seguintes níveis de evidência: Nível I: as evidências são provenientes de revisão sistemática ou metanálise de todos os relevantes ensaios clínicos randomizados controlados ou oriundos de diretrizes clínicas baseadas em revisões sistemáticas de ensaios clínicos randomizados controlados; Nível II: evidências derivadas de, pelo menos, um ensaio clínico randomizado controlado bem delineado; Nível III: evidências obtidas de ensaios clínicos bem delineados; Nível IV: evidências provenientes de estudos de coorte e de caso-controle bem delineados; Nível V: evidências originárias de revisão sistemática de estudos descritivos e qualitativos; Nível VI: evidências derivadas de um único estudo descritivo ou qualitativo; e Nível VII: evidências oriundas de opinião de autoridades e/ou relatório de comitês de especialistas.

Em seguida, os dados obtidos foram agrupados conforme o referencial teórico da tríade de Donabedian com núcleos temáticos na avaliação das maternidades ${ }^{(6)}$.

\section{RESULTADOS}

A pesquisa resultou em 17 artigos. Nove foram desenvolvidos no Brasil 
(52,9\%), dois em Uganda (11,7\%), e um estudo nos seguintes países: Escócia (5,8\%); Nova Zelândia (5,8\%); Indonésia (5,8\%), Senegal (5,8\%); Bangladesh, Haiti e Malawi (5,8\%); Tanzânia e Senegal (5,8\%); Nigéria (5,8\%).

Em relação ao ano, houve predomínio do ano de 2017 com sete artigos $(41,1 \%)$, seguido de 2008 com três (17,6\%), 2015 e 2014 com dois artigos cada (11,7\%) e os anos de 2018, 2016 e 2010 com um artigo (5,8\% cada). Quanto ao delineamento dos estudos, quatorze são estudos quantitativos $(82,3 \%)$ e três são pesquisas qualitativas (17,6\%). Dos estudos quantitativos, sete são coortes, quatro são transversais descritivos e dois inquéritos. Analisando o nível de evidência dos artigos, oito $(47,0 \%)$ possuíam nível $\mathrm{V}$, sete nível IV (41,1\%), um nível III $(5,8 \%)$ e um nível VI (5,8\%).

No tocante à avaliação da qualidade, seis estudos $(35,2 \%)$ avaliaram os serviços materno infantis de acordo com a estrutura, quatro $(23,5 \%)$ de acordo com os processos e três $(17,6 \%)$ utilizaram indicadores de resultados. Quatro artigos (23,5\%) usaram a percepção das mulheres como uma forma de avaliar as maternidades.

A maioria dos estudos atribuiu a estrutura como forma de avaliação e tinham foco na qualidade da assistência ao recém-nascido e redução da mortalidade

Quadro 1 - Caracterização da produção científica sobre a avaliação de maternidades nas dimensões de estrutura, processos e resultados. Não seria quadro a apresentação descrita???

\begin{tabular}{|c|c|c|c|}
\hline INDICADORES & AUTORES/ANO & MÉTODOS & VARIÁVEIS UTILIZADAS NA AVALIAÇÃO \\
\hline \multirow{6}{*}{ Estrutura } & Almeida et al/2008 & Estudo transversal & $\begin{array}{l}\text { Recursos humanos e equipamentos necessários para } \\
\text { reanimação neonatal. }\end{array}$ \\
\hline & Silva et al/2010 & Estudo transversal & $\begin{array}{l}\text { UTI neonatal e adulto, atividade de ensino, referência para } \\
\text { alto risco e volume de parto. }\end{array}$ \\
\hline & Bittencourt et al/2014 & $\begin{array}{l}\text { Coorte de base hospitalar } \\
\text { Nascer no Brasil }\end{array}$ & $\begin{array}{l}\text { UTI materna e neonatal, equipamentos, setores de apoio } \\
\text { e medicamentos, referência para alto risco, gestores } \\
\text { capacitados. }\end{array}$ \\
\hline & Bittencourt et al/2015 & $\begin{array}{l}\text { Coorte de base hospitalar } \\
\text { Nascer no Brasil }\end{array}$ & Recursos humanos, medicamentos, equipamentos. \\
\hline & Bittencourt et al/2016 & $\begin{array}{l}\text { Estudo transversal, nacional } \\
\text { de base hospitalar. }\end{array}$ & $\begin{array}{l}\text { Recursos humanos, medicações, equipamentos para aten- } \\
\text { dimento emergencial. }\end{array}$ \\
\hline & Winter et al/2017 & $\begin{array}{l}\text { Inquérito em Bangladesh, Hai- } \\
\text { ti Malawi, Senegal e Tanzânia }\end{array}$ & $\begin{array}{l}\text { Água corrente, eletricidade, privacidade, equipamentos, } \\
\text { medicamentos, treinamento de pessoal e supervisão. }\end{array}$ \\
\hline \multirow{4}{*}{ Processos } & Faye et al/2004 & Estudo transversal & $\begin{array}{l}\text { Avaliação de sinais vitais maternos e fetais anteparto e } \\
\text { pós-parto. }\end{array}$ \\
\hline & Oliveira et al/2008 & Estudo transversal & $\begin{array}{c}\text { Avaliação obstétrica e fetal, indicadores de boas práticas, } \\
\text { presença de acompanhante. }\end{array}$ \\
\hline & Leal et al/2008 & Estudo transversal & $\begin{array}{l}\text { Número de consultas de pré-natal, peso, realização de } \\
\text { exames e entrega em tempo oportuno e peregrinação. }\end{array}$ \\
\hline & Mawarti, Utarini/2017 & Coorte retrospectiva & $\begin{array}{l}\text { Tempo de espera na triagem, uso de ocitocina, medica- } \\
\text { mentos e tipo de parto. }\end{array}$ \\
\hline \multirow{3}{*}{ Resultados } & Barros et al/2008 & Estudo de coorte & $\begin{array}{l}\text { Apgar, hemorragia, admissão em unidade neonatal, peso } \\
\text { ao nascer, morbimortalidade. }\end{array}$ \\
\hline & Denham, et al/ 2017 & Estudo transversal & Trauma perineal, transferência, peso ao nascer e cesariana. \\
\hline & Grigg et al/2017 & Estudo transversal & $\begin{array}{l}\text { Apgar, hemorragia, admissão em unidade neonatal, peso } \\
\text { ao nascer, morbimortalidade. }\end{array}$ \\
\hline \multirow{4}{*}{$\begin{array}{l}\text { Perspectivas } \\
\text { das mulheres }\end{array}$} & Okonofua et al/2017 & Discussões de grupos focais & Satisfação com a qualidade no atendimento, \\
\hline & Kyaddondo et al/2017 & Grupos focais com puérperas & $\begin{array}{l}\text { Sobrevivência do recém-nascido, competência das partei- } \\
\text { ras e equipamentos. }\end{array}$ \\
\hline & Bohren et al/2017 & Grupos focais com gestantes & $\begin{array}{l}\text { Comunicação eficaz, respeito, dignidade e recursos huma- } \\
\text { nos. }\end{array}$ \\
\hline & Almeida et al/2018 & Estudo transversal & Acessibilidade e relação paciente/profissionais de saúde. \\
\hline
\end{tabular}


neonatal por meio de domínios como infraestrutura adequada, disponibilidade de equipamentos, medicamentos e recursos humanos capacitados ${ }^{(3,10-14)}$.

\section{DISCUSSÃO}

Esta revisão integrativa investigou a produção científica relacionada à avaliação de maternidades de acordo com critérios de estrutura, processos e resultados. A maior parte dos estudos selecionados avaliou a qualidade dos serviços de acordo com a estrutura das maternidades. Os elementos de estrutura e processos estão estreitamente ligados à qualidade dos serviços de saúde, em especial aos serviços de atenção obstétrica e neonatal ${ }^{(3)}$. O termo qualidade não foi utilizado como descritor nessa busca bibliográfica, o que poderia ter tornado a "chave de pesquisa" mais eficaz na busca primária e na fase final de inclusão dos artigos.

O modelo sistêmico proposto por Donabedian tem sido um método confiável para avaliar os serviços de atenção materno infantil, ora utilizados isoladamente, ora articulando seus elementos ${ }^{(6)}$.

Estudos têm demonstrado que o acesso a locais seguros para o parto e nascimento, assistência de profissionais qualificados e disponibilidade de estrutura e equipamentos adequados reduzem a mortalidade materna e neonatal ${ }^{(7)}$. Mas embora a relação isolada entre a estrutura e alguns indicadores perinatais possa indicar bom desempenho do serviço de saúde, não se pode garantir que ele seja de qualidade sem avaliar outros aspectos relevantes ligados à assistência oferecida ao binômio mãe-bebêt $\hat{e}^{(3)}$.

Por outro lado, a avaliação a partir de processos assistenciais utilizada como metodologia merece destaque uma vez que avalia as maternidades por meio das atividades desenvolvidas por profissionais de saúde e gestores para um atendimento obstétrico e neonatal adequado desde a internação até a alta. ${ }^{(8)}$

Evidências mostram que a melhoria nos cuidados prestados nas maternidades diminui as taxas de morbimortalidade
O tempo de

atendimento, a

classificação de

risco adequada, a

administração de

drogas em tempo

oportuno em

casos de urgência

e emergência

obstétricas e o uso

de tecnologias

apropriadas no

parto e nascimento

são indicadores que

refletem a qualidade

do serviço. Portanto,

os cuidados devem

ser seguros, eficazes,

oportunos, eficientes

e centrados nas

pacientes materna e neonatal. O tempo de atendimento, a classificação de risco adequada, a administração de drogas em tempo oportuno em casos de urgência e emergência obstétricas e o uso de tecnologias apropriadas no parto e nascimento são indicadores que refletem a qualidade do serviço. Portanto, os cuidados devem ser seguros, eficazes, oportunos, eficientes e centrados nas pacientes ${ }^{(9,10)}$

A preocupação com a qualidade no nascimento tem ganhado relevância, especialmente no Brasil, devido à persistência dos maus indicadores perinatais mesmo com a universalização hospitalar do parto e assistência realizada por médicos em $90 \%$ dos nascimentos. Em um cenário nacional marcado pelo excesso de intervenções desnecessárias e pela violência no momento do parto, recorde mundial de cesarianas; mortes maternas, fetais e neonatais evitáveis; torna-se imprescindível avaliar os processos de trabalho para uma assistência segura no parto e nascimento ${ }^{(11)}$.

Os indicadores de resultados são comumente utilizados por municípios, estados e países para medir o funcionamento dos serviços de saúde, em especial os estabelecimentos materno infantis que atendem partos. No Brasil, o monitoramento de indicadores perinatais estimulados pelas agendas e compromissos internacionais tornou-se obrigatório e está vinculado à liberação de investimentos e recursos financeiros. Sendo assim, os dados analisados nesse estudo utilizaram indicadores de resultados para comparar a segurança e eficácia entre centros de atendimentos materno infantis na assistência ao parto. ${ }^{(12,13)}$.

Descrever as experiências e expectativas das mulheres em relação ao seu parto foi citado em quatro estudos desta revisão. Os artigos aqui selecionados deram voz às mulheres que traduzem a sua avaliação na forma de atitudes respeitosas, cuidado qualificado e local seguro e com estrutura adequada para terem seus filhos em boas condições. Embora a Organização Mundial da Saúde tenha publicado recentemente o seu manual de experiências positivas no parto, grande parte das 
mulheres no mundo não tem assistência de profissionais qualificados durante o nascimento. Ademais, a satisfação dos usuários também é uma forma de avaliação e representa uma maneira de melhorar a qualidade dos serviços de saúde e discutir o cuidado ao paciente ${ }^{(14,15)}$.

Portanto, torna-se necessário propor novos estudos que explorem resultados advindos de diferentes fontes de dados e metodologias para melhor avaliar a qualidade dos serviços obstétricos e neonatais, proporcionando uma visão ampliada da atenção à saúde ofertada às
... proporcionando

uma visão ampliada

da atenção à saúde

ofertada às mulheres

e recém-nascidos em

seus locais de parto e

nascimento. mulheres e recém-nascidos em seus locais de parto e nascimento.

\section{CONCLUSÃO}

A síntese dos estudos analisados mostra que a avaliação das maternidades pode ser feita de forma multidimensional e ainda permanece como um desafio para os avaliadores. No entanto, não há uma definição de métodos específicos que expressem maior solidez para a avaliação de maternidades, ou ainda, que elementos de estrutura, processos ou resultados se sobressaiam entre si durante uma avaliação. -

\section{REFERÊNCIAS}

1. Rodrigues DP, Alves VH, Vieira RS, Leão DCMR, Paula ED, Pimentel MM. A violência obstétrica no contexto do parto e nascimento. Rev Enferm UFPE on line. 2018; 12(1):236-46. doi: https://doi.org/10.5205/1981-8963v12i01a23523p236-246-2018

2. Diniz SG, Salgado HO, Amdrezzo HFA, Carvalho PGC, Carvalho PCA, Aguiar CA, et al. Abuse and disrespect in childbirth care as a public health issue in Brazil: origins, definitions, impacts on maternal health, and proposals for its prevention. Rev Bras Crescimento Desenvolv Hum. 2015; 25(3):377-84. doi: http:// dx.doi.org/10.7322/jhgd.106080

3. Leal MC, Theme-Filha MM, Moura EC, Cecatti JG, Santos LMP. Atenção ao pré-natal e parto em mulheres usuárias do sistema público de sáude residentes na Amazônia Legal e no Nordeste, Brasil 2010. Rev Bras Saúde Matern Infant. 2015; 15(1):91-104. doi: http://dx.doi.org/10.1590/S1519-38292015000100008

4. Kyaddondo D, Mugerwa K, Byamugisha J, Oladapo OT, Bohren MA. Expectations and needs of Ugandan women for improved quality of childbirth care in health facilities: A qualitative study. International J Ginecol Obstetr. 2017; 139:38-46. doi: https:// doi.org/10.1002/ijgo.12405

5. Winter R, Yourkavitch J, Wang W, Mallick L. Assessment of health facility capacity to provide newborn care in Bangladesh, Haiti, Malawi, Senegal and Tanzania. J Glob Health. 2017; 7(2). doi: https://doi.org/10.7189/jogh.07.020509

6. Donabedian A. The definition of quality: a conceptual exploration. Explorations in quality assessment and monitoring. Michigan: Health Administration Press, 1988.

7. Bittencourt DAS, Gurgel RQ, Menezes MAS, Bastos LS, Leal MC. Neonatal care in Brazil: hospital structure and adequacy according to newborn obstetric risk. Paediatr Int Child Health. 2015; 35(3):206-12. doi: https://doi.org/10.1179/204690551 $5 Y .0000000028$

8. Bittencourt DAS, Domingues RMSM, Reis LNC, Ramos MM, Leal MC. Adequacy of public maternal care services in Brazil. Reprod Health. 2016; 13(3):120. doi: https://doi.org/10.1186/ s12978-016-0229-6

9. Bohren MA, Titiloye MA, Kyaddondo D, Hunter EC, Oladapo OT, Tunçalp O, et al. Defining quality of care during childbirth from the perspectives of Nigerian and Ugandan women: A qualitative study. International J Gynecol Obstetr. 2017; 139(1):4-16. doi: https://doi.org/10.1002/ijgo.12378

10. Mawarti $Y$, Utarini A, Hakimi M. Maternal care quality in near miss and maternal mortality in an academic public tertiary hospital in Yogyakarta, Indonesia: a retrospective cohort study. BMC Pregnancy Childbirth. 2017; 17(1):149. doi: https:// doi.org/10.1186/s12884-017-1326-4

11. Leal MC, Pereira APE, Domingues RMSM, Theme-Filha MM, Dias MAB, Pereira NM, et al. Intervenções obstétricas durante o trabalho de parto e parto em mulheres brasileiras de risco habitual. Cad Saúde Pública. 2014; 30(1):17-32. doi: https://doi. org/10.1590/0102-311X00151513

12. Denham S, Humphrey T, Taylor R. Quality of care provided in two Scottish rural community maternity units: a retrospective case review. BMC Pregnancy Childbirth 2017; 17(1):198. doi: https://doi.org/10.1186/s12884-017-1374-9

13. Grigg CP, Tracy SK, Tracy M, Daellenbach $R$, Kensington $M$, Monk $A$, et al. Evaluating Maternity Units: a prospective cohort study of freestanding midwife-led primary maternity units in New Zealand-clinical outcomes. BMJ open. 2017; 7(8). doi: https://doi.org/10.1136/bmjopen-2017-016288

14. Almeida KJQ, Roure FN, Bittencourt RJ, Santos RMDB, Bittencourt FV, Gottems LBBD, et al. Ouvidoria ativa em saúde: avaliação da qualidade da atenção ao parto e nascimento. Rev Saúde Pública. 2018; 52-76. doi: https://doi.org/10.11606/ S1518-8787.2018052017291

15. Okonofua F, Ogu R, Agholor K, Okike O, Abdus-salam R, Gana M, et al. Qualitative assessment of women's satisfaction with maternal health care in referral hospitals in Nigeria. Reprod Health. 2017; 14(44). doi: https://doi.org/10.1186/s129 78-017-0305-6 